\title{
Current Evaluation of Sepsis among Patients with Liver Cirrhosis
}

\author{
Elsayed Saad Abd Elbaser, MD and Olfat Abdel-Monem Ibrahim* \\ Tropical Medicine and Anaesthesia* Departments, Faculty of Medicine, Zagazig University, \\ Zagazig, Egypt
}

Corresponding Author Elsayed Saad Abd Elbaser MD

Mobile: 00201094986320

E mail: dr.sayedsaad79@gmai l.com

Key words: sepsis, cirrhosis, infection
Background and study aim: patients with liver cirrhosis have high incidence of sepsis. Spontaneous bacterial peritonitis and urinary tract infections are the most common infections among patients with liver cirrhosis. New criteria including quick qSOFA and sepsis-3 criteria are used for diagnosing sepsis in patients with liver cirrhosis. These criteria appear to be more accurate than SIRS. The aim of this study is to evaluate the existing scoring systems in our patients with liver cirrhosis to identify patients with sepsis.

Patients and Methods: This prospective study included 288 consecutive patients previously diagnosed to have liver cirrhosis and suspected to have bacterial/fungal infections. Quick Sequential (sepsis-related)

\section{INTRODUCTION}

Patients with decompensated cirrhosis have high incidence of sepsis. The prevalence of sepsis is about $30-50 \%$ in patients hospitalized for acute hepatic decompensation [1]. Spontaneous bacterial peritonitis and urinary tract infection are the most common infections among patients with cirrhosis, followed by chest infection, cellulitis and spontaneous bacteremia [2]. These different types of infections induce excessive systemic inflammation that may lead to decompensation, organ failure and acute-on-chronic liver failure in patients with cirrhosis [3].

Diagnosis of sepsis is still challenging in general population. There is no standard diagnostic test that allows easy and accurate diagnosis of sepsis [4]. Systemic inflammatory response syndrome (SIRS) criteria (at least two of the following: body temperature $<36^{\circ} \mathrm{C}$ or $>38^{\circ} \mathrm{C}$; heart rate $>90 \mathrm{bpm}$, respiratory rate $>20 / \mathrm{min}$, white blood organ failure assessment (qSOFA) criteria and sepsis-3 criteria were used to identify patients with organ dysfunction due to sepsis.

Results: qSOFA and sepsis-3 criteria are more accurate than SIRS in detecting sepsis among patients with cirrhosis (The area under the receiver operating characteristic curve (AUROC) value for a model with qSOFA and sepsis-3 was AUROC: 0.77 and 0.76), while AUROC for SIRS was 0.66 .

Conclusion: Sepsis-3 and qSOFA are more accurate than SIRS criteria in early detection of sepsis among patients with cirrhosis. Patients with positive criteria need intensive management due to high risk of in-hospital mortality.

cells $[\mathrm{WBC}]<4.000 / \mu \mathrm{L}$ or $>12.000 / \mu \mathrm{L}$ or immature neutrophils $>10 \%$ ) were used for diagnosing sepsis in cirrhosis [5]. However, it may be difficult to prove infection in addition to the previous criteria or the results are delayed. Also, patients with cirrhosis may have leucopenia because of hypersplenism, tachypnea because of hepatic encephalopathy or ascites, and/ or bradycardia because of the use of beta-blockers which make the clinical judge is more difficult [1]. Thus, SIRS criteria are ineffective for diagnosing sepsis in cirrhosis. Recently, organ dysfunction due to sepsis is defined as a change in sequential organ failure assessment (SOFA) score $\geq 2$ points or positive quick SOFA (at least two of the following: alteration in mental status, systolic blood pressure $\leq 100$ $\mathrm{mm} \mathrm{Hg}$ or respiratory rate $\geq 22 / \mathrm{min}$ ). Both scores are used recently instead of systemic inflammatory response syndrome (SIRS) criteria [5]. Both Sepsis-3 criteria and qSOFA were 
shown to be more accurate than SIRS criteria in predicting in-hospital mortality in patients with cirrhosis and bacterial infections [6]. As rapid diagnosis of sepsis in cirrhotic patients is a critical issue, there is a need for diagnostic scoring systems to facilitate early detection of sepsis. So, we aimed in this study to evaluate the existing scoring systems in our patients with liver cirrhosis to identify patients with sepsis in the context of available clinical and laboratory data.

\section{PATIENTS AND METHODS}

This prospective study included 288 consecutive patients previously diagnosed to have liver cirrhosis and suspected to have bacterial/fungal infections. They were selected among patients with cirrhosis were admitted to Intensive Care Unit (ICU) of tropical medicine and anaesthia departments, Zagazig University hospitals during the period between January 2018 and May 2018. Variables for sepsis criteria were calculated within $24 \mathrm{~h}$ after the admission. Quick Sequential (sepsis-related) organ failure assessment (qSOFA) criteria and sepsis-3 criteria were used to identify patients with organ dysfunction due to sepsis. Baseline SOFA was assessed using preadmission data. Acute changes in SOFA score of 2 points or more represent organ dysfunction.
Systemic screening for infections included all the followings :

1. Thorough history taking and clinical examination.

2. Complete blood counting.

3. Liver function including coagulation profile and kidney function tests.

4. Ascetic fluid sampling for total and differential leucocytes counting.

5. Urine analysis and measurement of urine output

6. Different body fluids culture (urine, blood, sputum, ascites) according to clinical setting.

7. Arterial blood gases (ABG) and electrocardiography (ECG)

8. Chest $\mathrm{x}$ ray

9. Ultrasonography on abdomen and pelvis.

After infection was suspected, patients were meticulously evaluated and promptly treated with empirical broad-spectrum antibiotic combination, depending on the site of infection, known colonization and previous antibiotic treatment. Antifungal therapy was added if fungal infection was suspected or documented. Antimicrobial treatment was narrowed after identification of the responsible pathogen. The patients were followed up until discharge or death.

Sequential [Sepsis-Related] Organ Failure Assessment Score :

\begin{tabular}{|l|c|c|c|c|c|}
\hline \multicolumn{1}{|c|}{ System } & $\mathbf{0}$ & $\mathbf{1}$ & $\mathbf{2}$ & $\mathbf{3}$ & $\mathbf{4}$ \\
\hline $\begin{array}{l}\text { PaO2/FIO2, } \\
\text { mmHg }\end{array}$ & $\begin{array}{c}\geq 400 \\
(53.3)\end{array}$ & $\begin{array}{c}<400 \\
(53.3)\end{array}$ & $<300(40)$ & $\begin{array}{c}<200(26.7) \text { with } \\
\text { respiratory support }\end{array}$ & $\begin{array}{c}<100 \text { (13.3) with } \\
\text { respiratory support }\end{array}$ \\
\hline Platelets $\times \mathbf{1 0 / u L}$ & $\geq 150$ & $<150$ & $<100$ & $<50$ & $<20$ \\
\hline Bilirubin, mg/dL & $<1.2$ & $1.2-1.9$ & $2-5.9$ & $6-11.9$ & $>12$ \\
\hline MAP & MAP $\geq 70$ & MAP<70 & $\begin{array}{c}\text { Dopamine }<5 \\
\text { or dobutamine } \\
\text { (any dose) }\end{array}$ & $\begin{array}{c}\text { Dopamine 5.1-15or } \\
\text { epinephrine } \leq 0.1 \text { or } \\
\text { norepinephrine } \leq 0.1\end{array}$ & $\begin{array}{c}\text { Dopamine }>15 \text { or } \\
\text { epinephrine }>0.1 \text { or } \\
\text { norepinephrine }>0.1\end{array}$ \\
\hline $\begin{array}{l}\text { Glasgow Coma } \\
\text { Scale score }\end{array}$ & 15 & $13-14$ & $10-12$ & $6-9$ & $<6$ \\
\hline Creatinine mg/dL & $<1.2$ & $1.2-1.9$ & $2-3.4$ & $3.5-4.9$ & $>5$ \\
\hline
\end{tabular}

\section{Statistical analysis :}

The SPSS version 16 was used for statistical analysis. Data were expressed as mean \pm standard deviation (SD) or number (\%) as appropriate. To identify the risk of mortality we used the area under the receiver operating characteristic (AUROC) curve.

\section{RESULTS}

A total of 432 patients with decompensated cirrhosis were admitted to ICU of tropical and anaesthia departments during the period of the study, among these patient 288 were suspected to have bacterial/fungal infections. Gender, age, the cause cirrhosis, the types of infection, positive cultures and type bacterial infection, all are represented in table (1). 
Overall, 16 patients (6\%) had 0 SIRS criteria, 81 patients (28\%) had 1, 125 patients (43\%) had 2, 50 patients (17\%) had 3 and 16 patients $(6 \%)$ had 4 with their characters of systemic inflammatory response (SIRS) criteria are shown in table (2). On the other hand, 56 patients $(20 \%)$ had 0 qSOFA score, 145 patients $(50 \%)$ had 1, 61 patients $(21 \%)$ had 2 and 26 patients $(9 \%)$ had 3 with their characters of qSOFA score are shown in table (3). Regarding sepsis-3 criteria, patients who have positive sepsis-3 criteria due to infection were 95 patients (33\%).

Regarding mortality, The number of patients who died was $3,8,13,13$, and 12 for $0,1,2,3$ and 4
SIRS criteria, while those of qSOFA are increased with higher scores 4, 11, 15 and 19 for $0,1,2$ and 3 qSOFA scores (Figs. 3,4). The mortality percentage in patients with SIRS criteria and qSOFA with 2 or more points is compared in table (4).

The area under the receiver operating characteristic curve (AUROC) value for a model with qSOFA was AUROC: 0.77 (95\%CI, 0.68-0.85) (Fig. 5) and that for sepsis-3 criteria was AUROC: 0.76 (CI; 0.68-0.85) (Fig. 6), while AUROC value for a model with SIRS criteria was lower AUROC: 0.66 (95\%CI, 0.57-0.75) (Fig. 7).

Table (1) : Demographic and clinical data

\begin{tabular}{|l|l|}
\hline \multicolumn{1}{|c|}{ Variables } & \multicolumn{1}{|c|}{ No (total: 288, \%) } \\
\hline Age & $\mathbf{6 1 . 5}(\mathbf{3 5 - 9 0 )}$ \\
\hline Gender & Male: $138(48 \%)$ \\
& Female: $150(52 \%)$ \\
\hline Cause of cirrhosis; & \\
Chronic hepatitis B & $30(10.5 \%)$ \\
Chronic hepatitis C & $159(55 \%)$ \\
Combined chronic B, C & $20(7 \%)$ \\
Undetermined & $79(27.5)$ \\
\hline Infection type; & UTI: $86(30 \%)$ \\
& SBP: $75(26 \%)$ \\
& Chest infections: $55(19 \%)$ \\
& Bacteremia: $29(10 \%)$ \\
& Cellulitis: $15(5 \%)$ \\
& Secondary peritonitis and rupture umbilical \\
& hernias: $28(10 \%)$ \\
\hline Positive cultures - no (\%) & $218(76 \%)$ \\
\hline No of bacteria per patient-n (\%); & \\
Monomicrobial & $160(56 \%)$ \\
Polymicrobial & $40(14 \%)$ \\
Culture negative & $70(24 \%)$ \\
Multi-drug resistant & $18(6 \%)$ \\
\hline
\end{tabular}

Table (2) : Characters of systemic inflammatory response (SIRS) criteria among the studied patients

\begin{tabular}{|l|l|}
\hline SIRS $\geq 2$ & $\mathbf{1 9 1}(66 \%)$ \\
\hline $\begin{array}{l}\text { 1- Temperature } \\
>38^{\circ} \mathrm{C} \text { or }<36^{\circ} \mathrm{C}\end{array}$ & $\mathbf{1 3 0}(\mathbf{4 5 \% )}$ \\
\hline 2- Heart rate $>\mathbf{9 0} \mathbf{b p m}$ & $\mathbf{1 5 9}(\mathbf{5 5 \% )}$ \\
\hline $\begin{array}{l}\text { 3- White blood cell count } \\
>12000 / \mu \mathrm{L} \text { or } \\
<4000 / \mu \mathrm{L} \text { or } \\
>10 \% \text { immature } \\
\text { Bands }\end{array}$ & $\mathbf{9 6}(\mathbf{3 3 \%})$ \\
\hline $\begin{array}{l}\text { 4- Respiratory rate } \\
>20 / \text { min or PaCO } 2 \\
<32 \text { mm Hg }\end{array}$ & \\
\hline
\end{tabular}


Table (3) : Characters of qSOFA model among the studied patients

\begin{tabular}{|l|l|}
\hline $\mathrm{qSOFA} \geq \mathbf{2}$ & $87(30 \%)$ \\
\hline Respiratory rate/min $\geq \mathbf{2 2 / m i n}$ & $70(24 \%)$ \\
\hline $\begin{array}{l}\text { Altered mental status, Glasgow coma scale } \\
\text { score } \leq \mathbf{1 3}\end{array}$ & $152(53 \%)$ \\
\hline Systolic blood pressure $\leq 100$ & $123(43 \%)$ \\
\hline
\end{tabular}

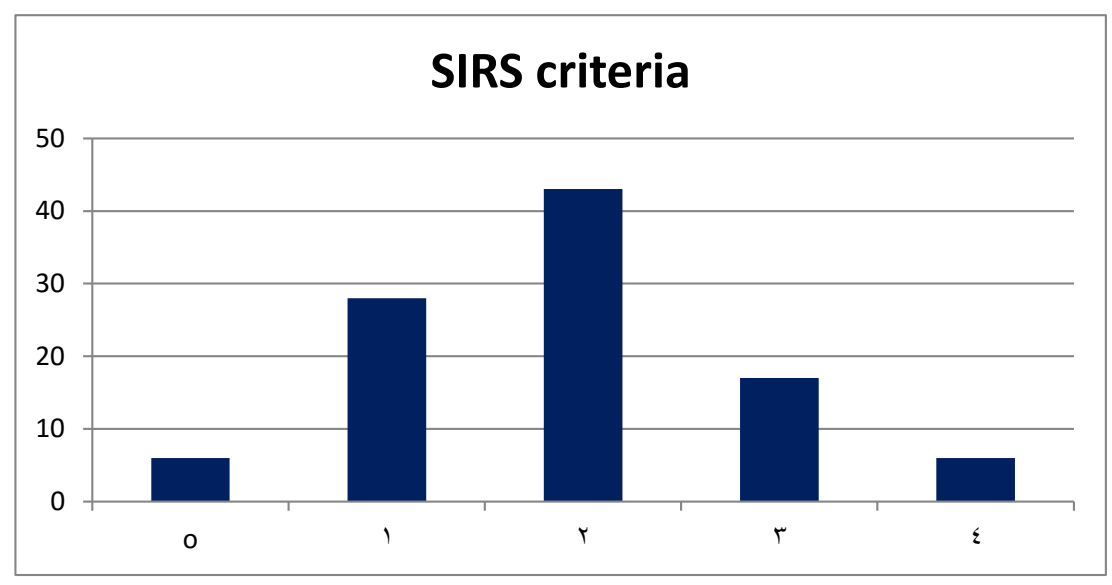

Figure (1) : Distribution of patients by SIRS criteria

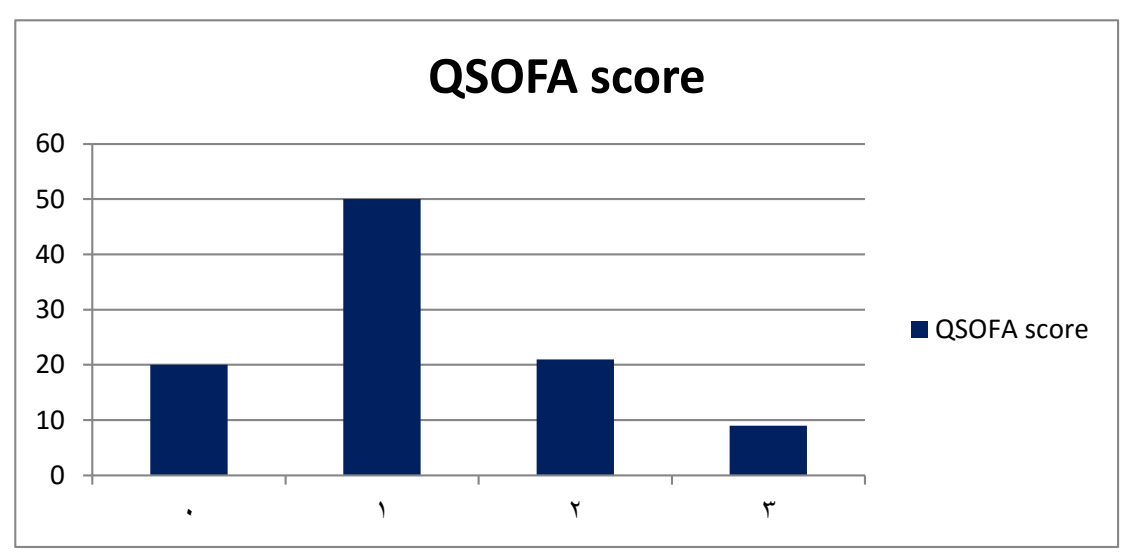

Figure (2) : Distribution of patients by qSOFA score.

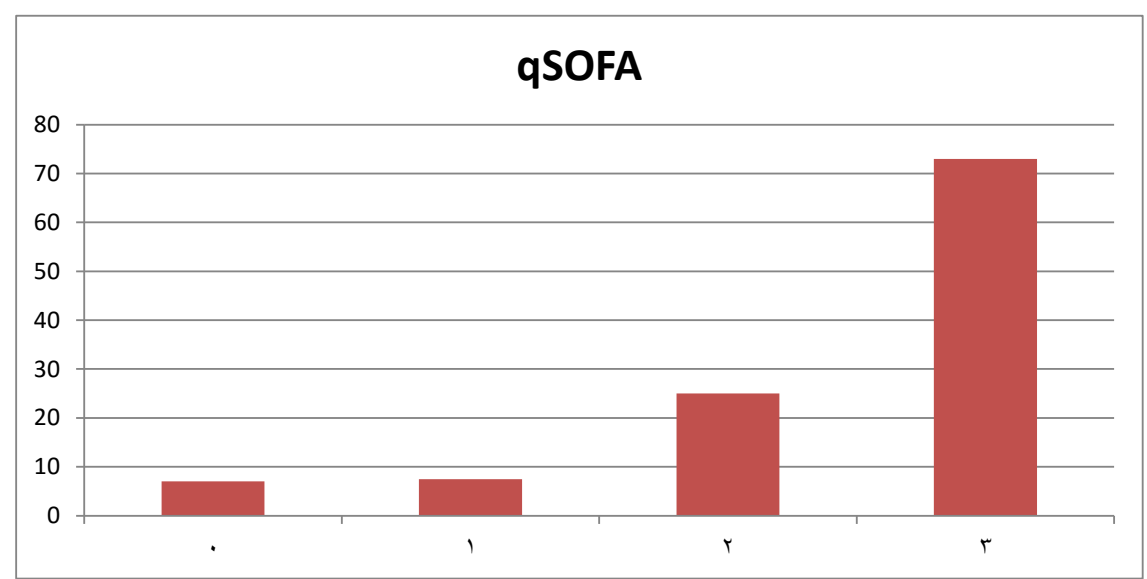

Figure (3) : Observed mortality rate according to qSOFA score

Abd Elbaser and Ibrahim, Afro-Egypt J Infect Endem Dis 2018; 8(4):182-188

https://aeji.journals.ekb.eg/

http://mis.zu.edu.eg/ajied/home.aspx 


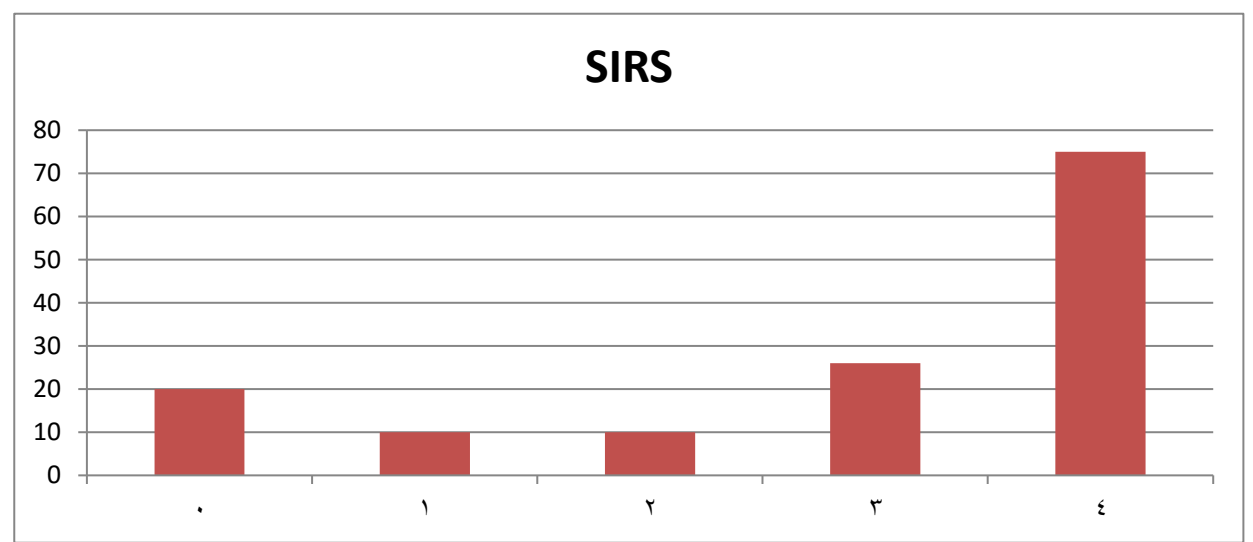

Figure (4) : Observed mortality rate according to SIRS criteria

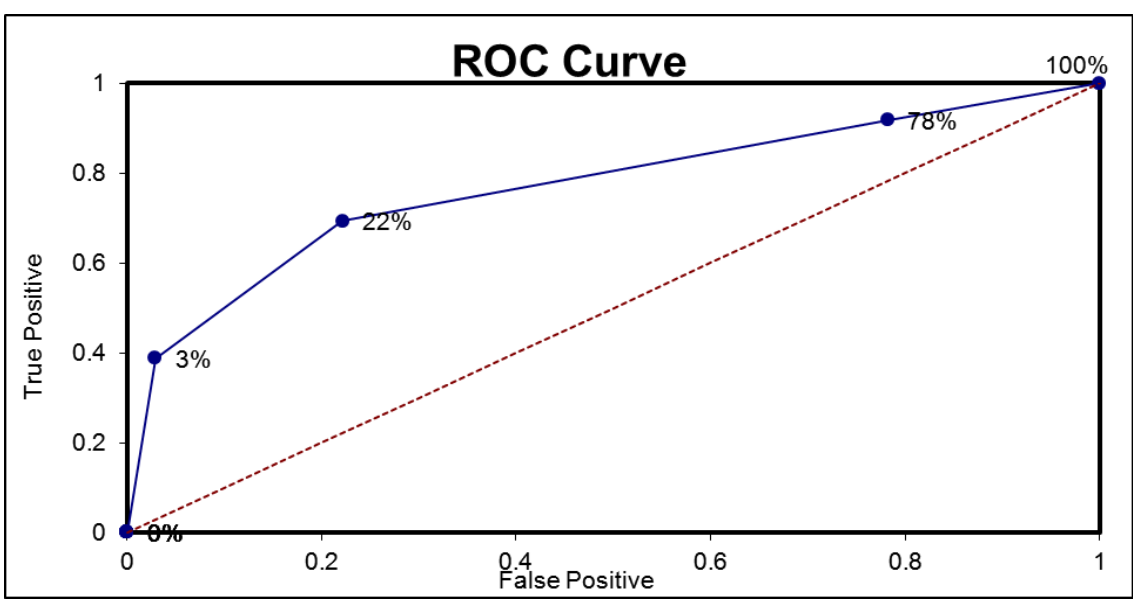

Figure (5) : Roc curve for qSOFA; AUROC:0.77 (95\%CI, 0.68-0.85)

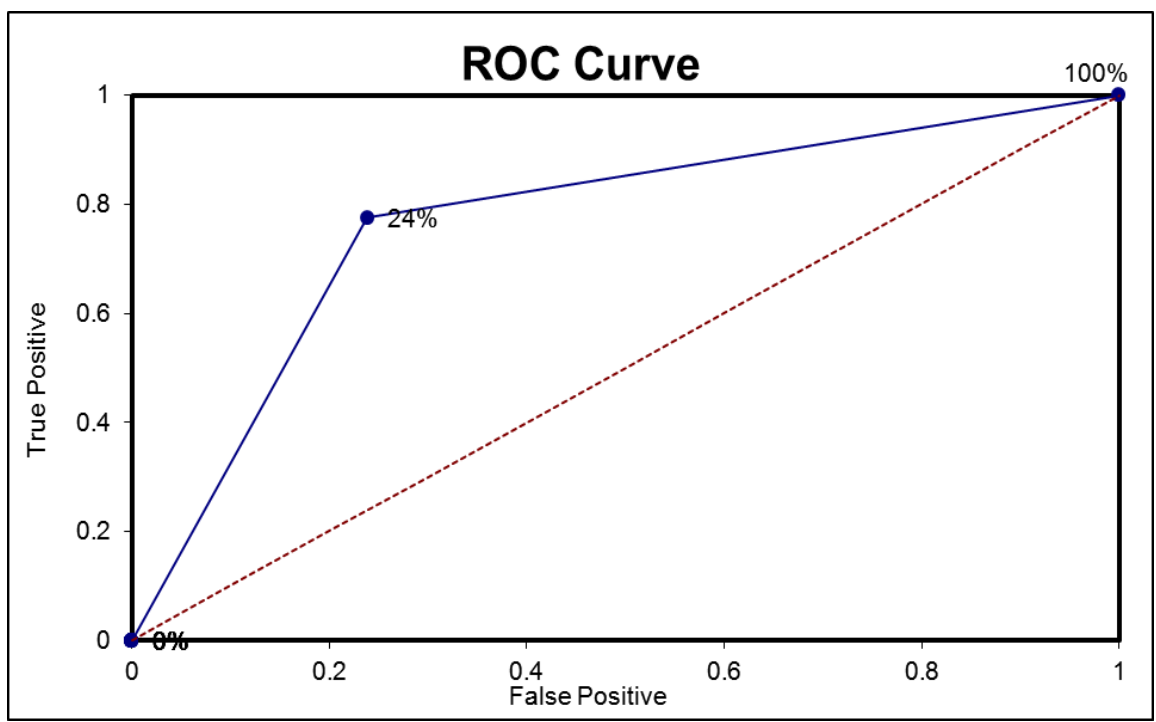

Figure (6) : ROC curve for sepsis3:AUROC:0.76(CI; 0.68-0.85)

Abd Elbaser and Ibrahim, Afro-Egypt J Infect Endem Dis 2018; 8(4):182-188 https://aeji.journals.ekb.eg/ 


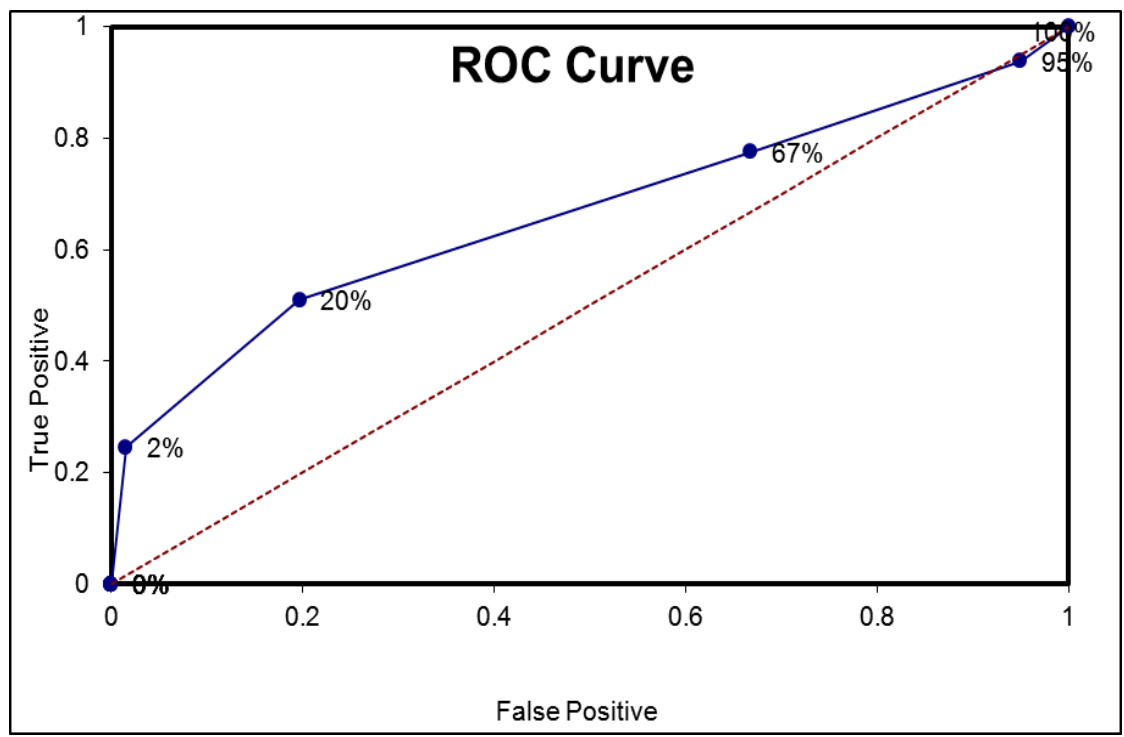

Figure (7) : ROC curve for SIRS; AUROC:0.66 (95\%CI, 0.57-0.75)

\section{DISCUSSION}

Sepsis is defined as life-threatening organ dysfunction due to a dysregulated host response to infection [4]. There is no gold standard test for diagnosis of sepsis but several clinical and laboratories data are used to identify patients with sepsis. Sepsis-3 criteria and qSOFA are widely used for prediction of sepsis in general population. However, their use in patients with cirrhosis is not yet adequately studied. Piano et al. [6] were the first to study the predictive validity of both sepsis-3 criteria and qSOFA in patients with cirrhosis and bacterial/fungal infections. They reported that both sepsis-3 criteria and qSOFA are superior to SIRS criteria in predicting severity of infection and mortality among patients with cirrhosis. Our study was aimed at evaluation of these criteria in identification of sepsis and to detect the predictive validity inhospital mortality in patients with cirrhosis.

The patients included in this study have decompensated cirrhosis based on clinical, laboratory and radiological data. The underlying cause of cirrhosis in most patients was chronic viral hepatitis (B and $\mathrm{C}$ ), due to its high prevalence in the Egyptian community. Urinary tract infection and spontaneous bacterial peritonitis were the most common infections. The body fluid cultures were positive in $76 \%$ of patients, Sepsis-3 and qSOFA criteria were positive in $33 \%$ and $30 \%$ of patients respectively. In Piano et al. [7], the underlying cause of cirrhosis in most patients was alcohol. The cultures were positive in $57 \%$ of patients and qSOFA criteria were positive in $23 \%$ of patients. This discrimination may be attributed to high incidence of bacterial rather than fungal or viral infections in our cirrhotic patients (alcoholics are more immunecompromised).

Sepsis -3 and qSOFA criteria had significantly greater predictive validity for in-hospital mortality (area under the receiver operating characteristic curve $($ AUROC) $=0.768$ and 0.770 respectively) than SIRS (AUROC=663). These results are consistent with that observed by Piano et al. [7]. AUROC for sepsis-3 and qSOFA are 0.784 and 0.732 respectively, while AUROC for sepsis was 0.606 . This high discrimination validity of both sepsis-3 and qSOFA criteria allow the use of these criteria as a bed side tool for early detection of cirrhotic patients with poor outcomes and hence apply more intensive management. Although high validity of both scores in detecting high risk patients, the need for objective laboratory markers may confer better detection of sepsis. So, further studies focusing on laboratory markers are needed.

\section{CONCLUSION}

Sepsis-3 and qSOFA are more accurate than SIRS criteria in early detection of sepsis among patients with cirrhosis. Patients with positive criteria need intensive management due to high risk of in-hospital mortality.

Acknowledgement: The authors would thank all colleagues who helped in conducting this study. Particular thanks to DR. Hala Ismail Mohammed for her great efforts. 
Ethical approval: The study protocol was reviewed and approved by the Institutional Review Board of the faculty of Medicine, Zagazig University, Egypt.

\section{Conflict of interest: None}

\section{Funding: None}

\section{REFERENCES}

1- Jalan R, Fernandez J, Wiest R, Schnabl B, Moreau R, Angeli P. et al. Bacterial infections in cirrhosis: a position statement based on the EASL Special Conference 2013. J Hepatol. 2014; 60: 1310-1324.

2- Fernández J, Acevedo J, Castro M, Garcia O, de Lope C, Roca D, et al. Prevalence and risk factors of infections by multi-resistant bacteria in cirrhosis: a prospective study Hepatology. 2012; 55: 1551-1561

3- Bernardi M, Moreau R, Angeli P, Schnabl B, Arroyo V. Mechanisms of decompensation and organ failure in cirrhosis: from peripheral arterial vasodilation to systemic inflammation hypothesis. $J$ Hepatol. 2015; 63: 1272- 1284

4- Singer M, Deutschman C, Seymour C, ShankarHari M, Annane,D, Bauer M. et al. The Third International Consensus Definitions for Sepsis and Septic Shock (Sepsis-3). JAMA. 2016; 315:801-810.

5- Fernández J, Gustot T. Management of bacterial infections in cirrhosis. J Hepatol. 2012;56:1 - 12

6- Piano S, Bartoletti M, Tonon M, Baldassarre M, Chies G, Romano A. et al. Assessment of Sepsis3 Criteria and quick SOFA in patients with cirrhosis and bacterial infections. Gut. 2018; 67 (10):1892-1899

7- Piano S, Singh V, Caraceni P, Maiwall R, Alessandria C, Fernandez J. et al. Epidemiology, predictors and outcomes of multi drug resistant bacterial infections in patients with cirrhosis across the world. Final results of the global study. Digestive and Liver Disease 2018; 50 (15); 1-19. 